\title{
Life expectancy of diabetic patients undergoing vitreous surgery
}

\author{
Horst Helbig, Ulrich Kellner, Norbert Bornfeld, Michael H Foerster
}

\begin{abstract}
Background-Patients with advanced diabetic eye disease are commonly in poor general health. In addition to the ocular status, life expectancy is an important factor in the decision whether and how to perform vitreous surgery. The present study investigates mortality and risk factors for survival in diabetic patients following vitrectomy.

Methods-The follow up of 332 consecutive patients who underwent vitrectomy for complications of diabetic retinopathy between 1990 and 1994 was studied retrospectively. Survival and risk factors for survival were analysed using the KaplanMeier life table method and for multivariate analysis the Cox proportional hazard model.

Results-The 5 year survival rate was $68 \%$. Absence of heart disease was the most important predicting factor for survival. Fifty per cent of the patients with heart disease had died within 3.5 years. Patients without heart disease had a 5 year survival rate of $90 \%$. Other significant, independent risk factors were age and presence of nephropathy.

Conclusion-In diabetic patients undergoing vitrectomy the presence of heart disease indicates a poor prognosis for survival. This should be taken into consideration for indications and strategies in cases of vitrectomy.

(Br F Ophthalmol 1996;80:640-643)
\end{abstract}

Department of Ophthalmology, Universitätsklinikum Benjamin Franklin, Freie Universität Berlin, Berlin, Germany

H Helbig

U Kellner

N Bornfeld

M H Foerster

Correspondence to:

H Helbig, Department of

Ophthalmology,

Universitätsklinikum

Benjamin Franklin, Freie

Universität Berlin,

Hindenburgdamm 30, 12200

Berlin, Germany.

Accepted for publication

11 March 1996
Vitrectomy has become a standard treatment for complications of proliferative diabetic retinopathy. Many studies have investigated ocular factors which influence visual prognosis. Several indications for vitreous surgery have been defined. ${ }^{1}$ However, in a situation where a surgeon is considering a vitrectomy for an individual diabetic patient, other extraocular factors cannot be ignored. Patients with severe proliferative diabetic retinopathy suffer from a systemic disease, not only affecting the eye, but also kidney, heart, nerves, and other organs. Improvement of anaesthesia techniques allows us to operate safely even these patients in poor health. When proposing to operate on a diabetic eye, the estimation of the life expectancy of the patient is an essential factor for the decision whether and how to operate. Considering the importance to the clinician, there are only few analyses of this factor. ${ }^{2-4}$ The purpose of the present study was to provide data for life expectancy and risk factors for survival in diabetic patients undergoing vitrectomy.

\section{Patients and methods}

We have reviewed the records of 337 consecutive patients (420 operated eyes) undergoing vitreous surgery for complications of diabetic retinopathy between 1990 and 1994 operated by the authors. For patients who were operated on both eyes only the data at the time of the first vitrectomy were considered. Data on ocular and systemic factors at the time of surgery were recorded in a computer data base. Preoperatively, chest $x$ ray, ECG, and blood tests including serum creatinine were performed in all patients. All operations were performed under general anaesthesia with the patient in a stable situation of general health. No serious complications or other serious systemic complications occurred during surgery or in the immediate postoperative period.

Systemic factors recorded were age, sex, duration of diabetes, insulin treatment, age at diagnosis of diabetes, presence of heart disease (history of myocardial infarction, symptoms of heart failure upon clinical examination or chest $x$ ray, signs of congestion, signs of coronary heart disease on ECG), presence of arterial hypertension (antihypertensive medication or arterial blood pressure $\geqslant 160 / 95 \mathrm{~mm} \mathrm{Hg}$ ), serum creatinine, history of renal dialysis, history of toe or foot amputation, body mass index as a measure for overweight (body weight (kg) divided by height $\left(\mathrm{m}^{2}\right)$ ). Ocular factors recorded were presence of cataract, visual acuity at last follow up, presence of retinal detachment, and rubeosis of the iris at the time of surgery.

Follow up data were obtained between January 1995 and October 1995. If the charts did not provide sufficient follow up, the information was obtained from the referring ophthalmologist, from the patient or his relatives by telephone inquiry, or from the residents' registration office. The mean follow up time was 26 (SD 15) months. Data were analysed using the Kaplan-Meier life table method. For univariate analysis of risk factors cumulative survival was compared using the log rank test. For multivariate analysis of risk factors the Cox proportional hazard model was used.

\section{Results}

Five patients had moved abroad and could not be traced. These cases were excluded from the analysis. A total of 332 patients were further 


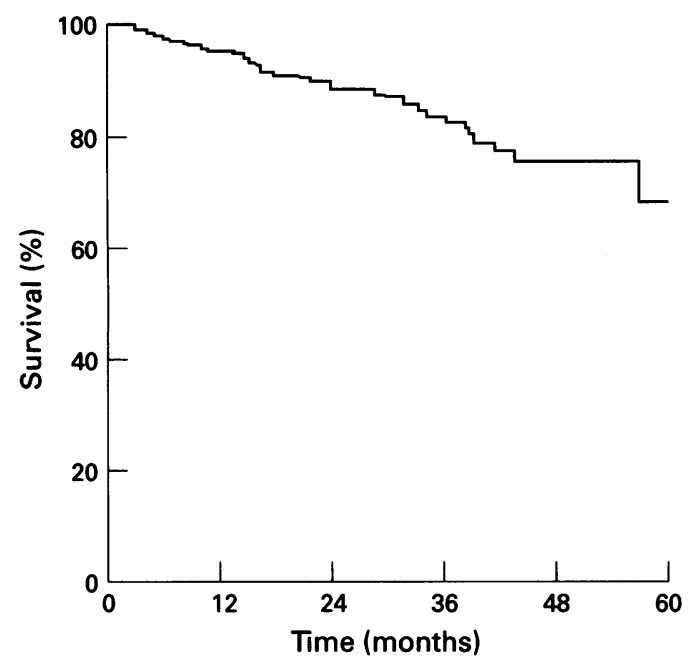

Figure 1 Kaplan-Meier analysis of survival of 332 patients after vitrectomy for diabetic retinopathy.

analysed: 47 patients had died. Cumulative survival is shown in Figure 1. The 3 year survival rate was 83 (SE 3) \%, and 5 year survival rate was $68(8) \%$.

A total of 153 patients were male and 179 were female. Ages ranged from 24 to 91 years with a median of 58 years (mean 56 (SD 14) years). The duration of diabetes ranged from one to 48 years with a median of 21 , and a mean 21 (9) years. In 114 patients diabetes had been diagnosed before age 30; in 218 patients after this age. A total of 276 patients were treated with insulin, 54 with oral antidiabetic drugs, and two only with diet. Arterial hypertension was present in 206 patients: 118 patients showed evidence of heart disease. Amputation of lower extremities had been performed in 31 patients: 13 patients were on renal dialysis. Serum creatinine was above 150 $\mu \mathrm{mol} / 1$ in 56 patients (including renal dialysis patients). Body mass index was above $28 \mathrm{~kg} / \mathrm{m}^{2}$ (indicating overweight) in 116 patients.

The lens was clear in 135 patients, the others had cataract of some degree or had had cataract surgery. Visual acuity at the last follow up visit was better than $1 / 50$ in 240 patients. Rubeosis of

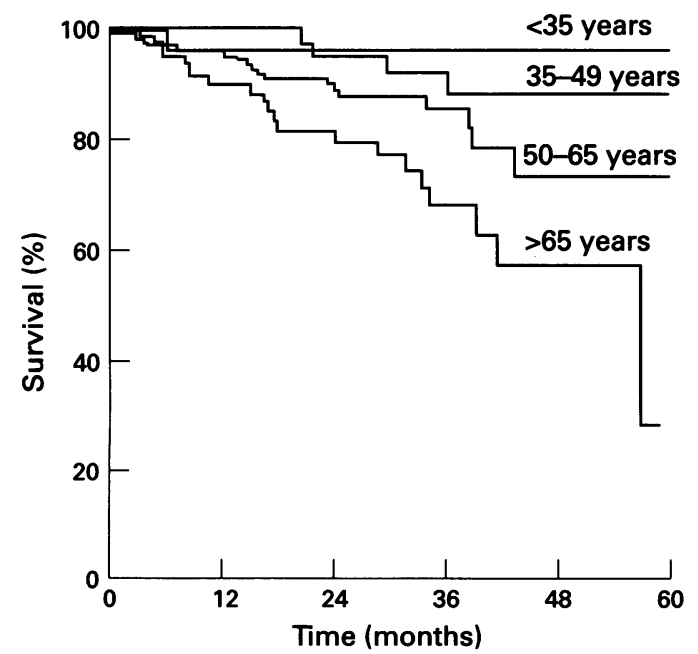

Figure 2 Kaplan-Meier analysis of survival after vitrectomy for diabetic retinopathy for different age groups. $n=28$ for age $<35$ years; $n=63$ for age $35-49 ; n=155$ for age $50-65 ; n=86$ for age $>65$ years $(p=0.002)$.

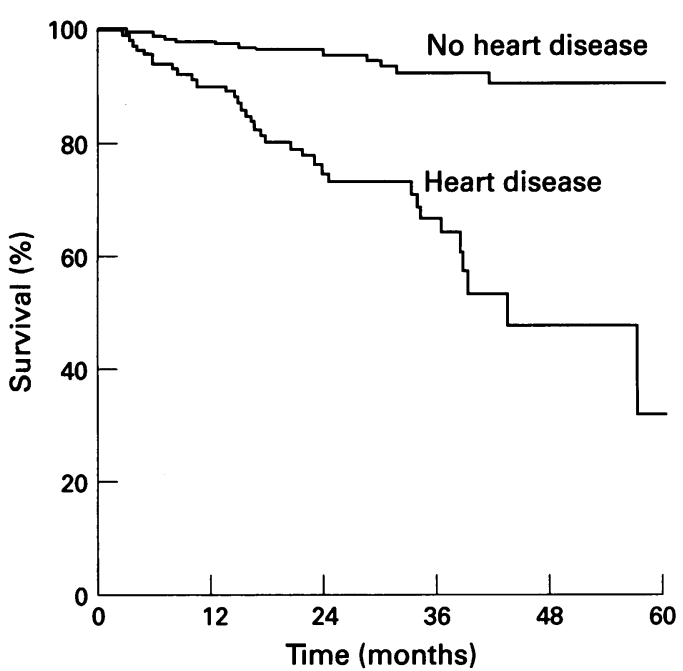

Figure 3 Kaplan-Meier analysis of survival after vitrectomy for diabetic retinopathy for patients with $(n=118)$ and without $(n=214)$ heart disease $(p<0.0001)$.

the iris was present in 91 eyes. The retina was detached preoperatively in 196 patients.

In univariate analyses the following factors were significantly associated with poorer survival after vitrectomy: higher age, age $>30$ years at diagnosis of diabetes, therapy with oral antidiabetic drugs, evidence of heart disease, and serum creatinine above $150 \mu \mathrm{mol} / \mathrm{l}$. If tested together with age in a Cox model, age at diagnosis of diabetes $>30$ years and therapy with oral antidiabetic drugs were no longer significant factors. The effect of age, heart disease, and renal failure on survival are shown in Figures 2-4. Results of a multivariate analysis using the Cox proportional hazard model including these three factors are shown in Table 1. The results indicate that age, heart disease and renal failure were statistically independent factors for survival and heart disease was the most important risk factor for survival.

If the subgroup of patients with a diagnosis of diabetes before age 30 (mainly type I diabetics) was tested separately for risk factors for survival, the only significant predictor was the presence of heart disease. Creatinine and age were not significant factors in type I diabetics.

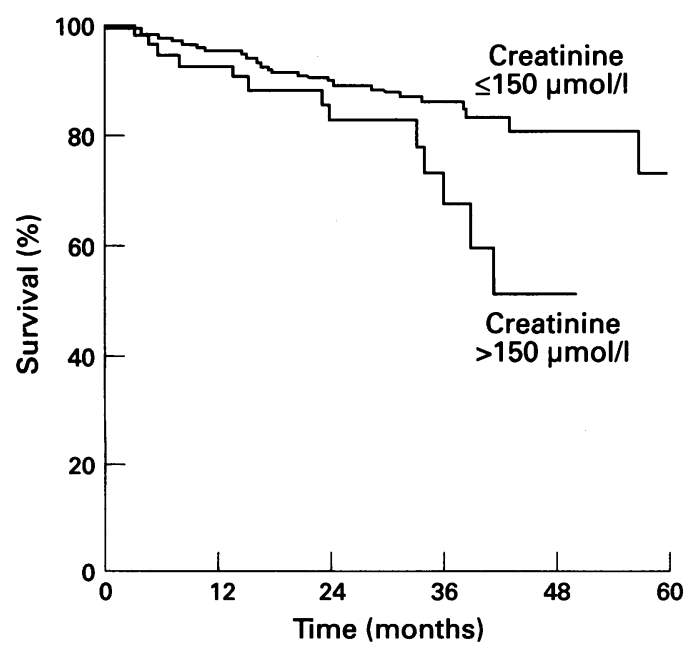

Figure 4 Kaplan-Meier analysis of survival after vitrectomy for diabetic retinopathy for patients with serum creatinine $>150 \mu \mathrm{mol} / \mathrm{l}(n=56)$ and $\leqslant 150 \mu \mathrm{mol} / \mathrm{l}(n=276$; $p=0.012$ ). 
Table 1 Results of a Cox proportional hazard model for factors influencing survival after vitrectomy for diabetic retinopathy

\begin{tabular}{lllr}
\hline & $\begin{array}{l}\text { Hazard } \\
\text { ratio }\end{array}$ & $\begin{array}{l}95 \% \\
\text { Confidence } \\
\text { limits }\end{array}$ & pValue \\
\hline $\begin{array}{l}\text { Age (years) } \\
\begin{array}{c}\text { Heart disease (present } \\
\text { versus absent) }\end{array}\end{array}$ & 1.03 & $1.0-11.06$ & 0.0078 \\
$\begin{array}{c}\text { Creatinine }(>150 \\
\text { versus } \leqslant 150 \mu \mathrm{mol} / \mathrm{l})\end{array}$ & 1.42 & $1.47-2.91$ & $<0.0001$ \\
\hline
\end{tabular}

In the younger onset diabetics the 3 year survival rate was 90 (3) \%. In the subgroup of patients with a diagnosis of diabetes after the age of 30 (mainly type II diabetics) the 3 year survival rate was 83 (3) \%. In this subgroup, the presence of heart disease, age, and creatinine levels above $150 \mu \mathrm{mol} / 1$ were significant independent predictors.

\section{Discussion}

Patients with diabetes mellitus still have a reduced life expectancy. The annual mortality risk for insulin dependent diabetic patients is more than 20 times greater ${ }^{5}$ and for noninsulin dependent diabetics approximately twice as high $^{6}$ compared with the general population.

Presence and severity of diabetic retinopathy is an indicator for an advanced disease stage and is associated with reduced survival. The 5 year survival rate for type I diabetics without retinopathy was $99 \%$ compared with only $76 \%$ for those with proliferative retinopathy. ${ }^{7}$ For type II diabetics the respective 5 year survival rate was $77 \%$ without retinopathy and $53 \%$ with proliferative retinopathy.

Diabetic patients undergoing vitrectomy suffer from advanced proliferative diabetic retinopathy. The 5 year postoperative survival rate in the present study was $68 \%$, similar to the published epidemiological data for mortality of patients with proliferative diabetic retinopathy. ${ }^{7}$ Other studies analysing survival following vitreous surgery in diabetic patients found 5 year survival rates of $73 \%,{ }^{8} 75 \%,{ }^{2} 80 \%,{ }^{3}$ and $85 \%,{ }^{4}$ but the mean age in our study was about 10 years higher than in most of the other studies. A 10 year survival rate after vitreous surgery of $50 \%$ has been reported. ${ }^{9}$

In large epidemiological studies of patients with diabetic retinopathy the main risk factors for survival, besides age, were renal failure and cardiovascular disease. ${ }^{7}$ Microalbuminuria has been shown to be related to cardiovascular mortality. ${ }^{10}$ Diabetics with coronary heart disease have a particularly poor prognosis. ${ }^{11}$ Studies on the survival of diabetic patients following vitrectomy have identified age, duration of diabetes, age at diagnosis of diabetes, and the presence of nephropathy ${ }^{2}$ as well as neuropathy ${ }^{4}$ as risk factors for survival. These studies have not included heart disease in their analyses. Cardiovascular disease is the most common cause of death in diabetic patients with about $75 \%$ ultimately dying of coronary heart disease. ${ }^{12}$ In our series the presence of heart disease was the main factor predicting survival. Fifty per cent of the patients with heart disease had died within 3.5 years while $90 \%$ of the patients without heart disease survived for more than 5 years. We also confirmed nephropathy as an independent risk factor for survival, but it was less important than heart disease.

The identification of heart disease as major risk factor for poor prognosis can have implications for indications and strategies of vitreous surgery. In many cases with diabetic eye disease the indications for surgery are not absolutely clearcut. In these cases the estimation of the life expectancy can help us to give the best advice to the patient. Surgery on an eye with active proliferative disease and useful vision has a beneficial long term effect but surgery also bears the risk of immediate vision loss. ${ }^{13} \mathrm{~A}$ patient with a life expectancy limited to few years may not benefit from vitrectomy in this situation. In contrast, for eyes with vitreous haemorrhage and attached retina the surgical risk is low. ${ }^{14}$ In this situation, especially in patients with a blind fellow eye, it is preferable to operate early to provide the best possible quality of life for the patient than to wait many months for spontaneous clearance of the media opacity. The indication for silicone as vitreous substitute should be more permissive in patients with reduced life expectancy. Silicone provides immediate visual rehabilitation, ${ }^{15}$ and the postoperative period of low vision due to haemorrhage or long acting gas tamponade can be avoided. For these patients silicone related late complications are less relevant.

On the other hand, diabetic patients without evidence of heart disease have an excellent prognosis with a 5 year survival rate of $90 \%$. These are the patients who will experience the long term benefits of vitreous surgery. ${ }^{9}$

1 Ho T, Smiddy WE, Flynn HJ. Vitrectomy in the management of diabetic eye disease. Surv Ophthalmol 1992;37:190-202.

2 Gollamudi SR, Smiddy WE, Schachat AP, Michels RG Vitale S. Long-term survival rate after vitreous surgery for complications of diabetic retinopathy. Ophthalmology 1991;
colte 98:18-22.

3 Summanen P, Karhunen U, Laatikainen L. Characteristics and survival of diabetic patients undergoing vitreous surgery. Acta Ophthalmol 1987;65:197-202.

4 Uchio E, Inamura $M$, Ohno S, Taguchi H, Saeki K. Survival rate after vitreous surgery in patients with diabetic retinopathy. Ophthalmologica 1993;206:83-88.

5 Dormann JS, Laporte RE, Kuller LH, Cruickshanks KJ, Orchard TJ, Wagener DK, et al. The Pittsburgh insulindependent diabetes mellitus (IDDM) morbidity and mortality study. Mortality results. Diabetes 1984;33:271morta
Panzram

6 Panzram G. Mortality and survival in type 2 (non-insulindependent) diabetes mellitus. Diabetologia 1987;30:123depend

7 Klein R, Moss SE, Klein BEK, DeMets DL. Relation of ocular and systemic factors to survival in diabetes. Arch Intern Med 1989;149:266-72.

8 Blankenship GW. Stability of pars plana vitrectomy results for diabetic retinopathy complications; a comparison of five-year and six-month postvitrectomy findings. Arch Ophthalmol 1981;99:1009-12.

9 Blankenship GW, Machemer R. Long-term diabetic vitrectomy results. Report of 10 year follow up. Ophthalmology 1985;92:503-6.

10 Deckert T, Poulsen JE, Larsen M. Prognosis of diabetics with diabetes onset before age 31. Diabetologia 1978;14: 363-70.

11 Ulvenstam G, Åberg A, Bergstrand R, Johansson S, Pennert $\mathrm{K}$, Vedin A, et al. Long-term prognosis after myocardial infarction in men with diabetes. Diabetes 1985;34:787-92.

12 Chait A, Bierman EL. Pathogenesis of macrovascular disease in diabetes. In: Kahn CR, Weir GC, eds. Foslin's diabetes mellitus. Philadelphia: Lea \& Febiger, 1994:648-64.

13 Diabetic Retinopathy Vitrectomy Study Research Group. Early vitrectomy for severe proliferative diabetic retinopa- 
thy in eyes with useful vision. Results of a randomized trial. Diabetic Retinopathy Vitrectomy Study Report 3. The Diabetic Retinopathy Vitrectomy Study Research Group. Ophthalmology 1988;95:1307-20.

14 Diabetic Retinopathy Vitrectomy Study Research Group Early vitrectomy for severe vitreous hemorrhage in diabetic retinopathy. Four-year results of a randomized trial. Diabetic Retinopathy Vitrectomy Study Report 5. Arch Ophthalmol 1990;108:958-64.

15 Lucke $\mathrm{K}$, Laqua H. Silicone oil in the treatment of complicated retinal detachments. Berlin, Heidelberg, New York: Springer Verlag, 1990. 